\title{
Retrospective Study of the Use of a Fractional Radio Frequency Ablative Device in the Treatment of Acne Vulgaris and Related Acne Scars
}

\author{
Judith Hellman \\ Mt. Sinai Hospital, New York, USA \\ Email: jhderm@gmail.com
}

Received 11 October 2015; accepted 5 December 2015; published 8 December 2015

Copyright (C) 2015 by author and Scientific Research Publishing Inc. This work is licensed under the Creative Commons Attribution International License (CC BY). http://creativecommons.org/licenses/by/4.0/

\begin{abstract}
Background: Acne vulgaris (AV) is a common disease that often results in disfiguring facial scarring that carries into adulthood. Here we report our experience with fractional radiofrequency (FRF) device in treatment of patients with acne and acne related scarring. Materials \& Methods: We retrospectively reviewed the charts of all patients with acne scarring who completed a four treatment regimen in our clinic. Results: We identified eight patients who completed four treatments with median age of 20.5 years (range 17 - 41). All patients demonstrated significant improvement of acne lesions and acne scarring. Skin biopsies demonstrated reduction of scar depth and increased new collagen production, and repopulation of the scar tissue by elastic fibers and adnexal structures after the fourth treatment. Conclusion: FRF emerges as a safe and effective treatment modality for $\mathrm{AV}$ and acne related scars. Further randomized controlled studies are required to fully evaluate the magnitude of this positive effect and more basic science studies are needed in order to better characterize its mechanism of action on acne lesions.
\end{abstract}

\section{Keywords}

Acne Vulgaris, Radiofrequency, Scarring

\section{Introduction}

Acne vulgaris (AV) is a disease afflicting mostly adolescents and young adults, often resulting in disfiguring facial scarring that carries into adulthood. While estimates on the prevalence of acne related scarring vary substan- 
tially [1], the psycho-social morbidity is significant [2] and therefore it is crucial to effectively treat AV in order to avoid scarring. The pathogenesis of acne relates to increased sebum production in the setting of aberrant follicular keratinization and propionibacterium acnes proliferation and significant perifollicular inflammation [3]. This process leads to local tissue damage resulting in activation of a wound healing cascade and imbalance in collagen deposition and matrix degradation may result in permanent scarring [4].

Current treatment regimens for acne scarring include chemical peels, dermabrasion, large and small surgical excisions, subcision, as well as various lasers, each with variable reports of success and advantages as well as limitations [5]. Radiofrequency (RF) devices represent novel technology that emerged just over a decade ago and is now used routinely for treatment of rhytides, cellulite, skin laxity and AV [6]. More recently fractional RF has been used to treat these cosmetic indications. However, the effect of fractional RF on acne related scarring has not been well characterized.

Here we present our experience with fractional RF (FRF) device in treatment of eight patients with acne and acne related scarring.

\section{Materials \& Methods}

Patients: all patients were informed about the risks, benefits and alternative treatments and all provided an informed consent based on the 1975 Declaration of Helsinki. Patients were generally young adults with history of acne scarring with or without active acne lesions. Patients with conditions considered to be contra-indicated per the manufacturer's instructions were not considered good candidates for therapy.

Treatments: we retrospectively reviewed the charts of all patients with acne scarring who completed a four treatment regimen. Each patient underwent four treatments, four weeks apart. Treatments involved only areas of the face. Pictures were taken immediately prior to the first treatment and after the fourth treatment. On average pictures were retaken 36 days after the fourth treatment (range 7 - 96 days). All patients received initial doses of $20-40 \mathrm{~mJ} / \mathrm{pin}$, and the doses were increased each visit, based on patient tolerance. All treated areas received one or two passes based on the treatment site and patient tolerance. Double and triple stacking of pulses was done as needed.

Prior to treatment the skin was cleansed with absolute ethanol. Treatments were performed on the affected areas, using either a regular or a coated 24 pin tip, depending on skin type. Two passes, with double or triple stacked pulses were performed with a third partial pass in the heavily scarred areas in a number of patients. Immediately following therapy, Aquaphor ${ }^{\mathrm{TM}}$ (Beiersdorf Inc. Wilton, CT) ointment was applied to the skin. Patients were instructed to continue using Aquaphor ${ }^{\mathrm{TM}}$ at home 2 - 3 times a day for a total of three days after the procedure. Concurrent treatments included topical agents (Dapsone or Clindamycin) twice a day. Patient demographics and treatment characteristics are summarized in Table 1.

Device: all patients were treated with an array of $24 \mathrm{RF}$ conducting needles, alternating current with two long side electrodes (Figure 1). Each needle is $2500 \mu \mathrm{m}$ long and $200 \times 300 \mu \mathrm{m}$ wide at the base. The coated needles are insulated along $2000 \mu \mathrm{m}$, leaving the distal $500 \mu \mathrm{m}$ uncoated. The hand piece was loaded to the Fractora ${ }^{\mathrm{TM}}$ platform (also applicable to InMode ${ }^{\mathrm{TM}}$ or BodyTite ${ }^{\mathrm{TM}}$ platforms), Invasix Ltd./InMode MD Ltd., Israel).

Table 1. Study participants and treatment characteristics.

\begin{tabular}{cccccc}
\hline Subject & Age (yrs.) & Sex & Skin type $^{*}$ & Treatment \# & Coated/uncoated \\
\hline $\mathbf{1}$ & 18 & M & I & 4 & uncoated \\
$\mathbf{2}$ & 41 & F & V & 4 & coated \\
$\mathbf{3}$ & 17 & F & IV & 4 & coated \\
$\mathbf{4}$ & 24 & F & IV & 4 & coated \\
$\mathbf{5}$ & 26 & F & III & 4 & uncoated \\
$\mathbf{6}$ & 18 & F & IV & 5 & coated \\
$\mathbf{7}$ & 19 & M & III & 4 & uncoated \\
$\mathbf{8}$ & 22 & F & II & 4 & uncoated \\
\hline
\end{tabular}

*Fitzpatrick skin type [10]. 


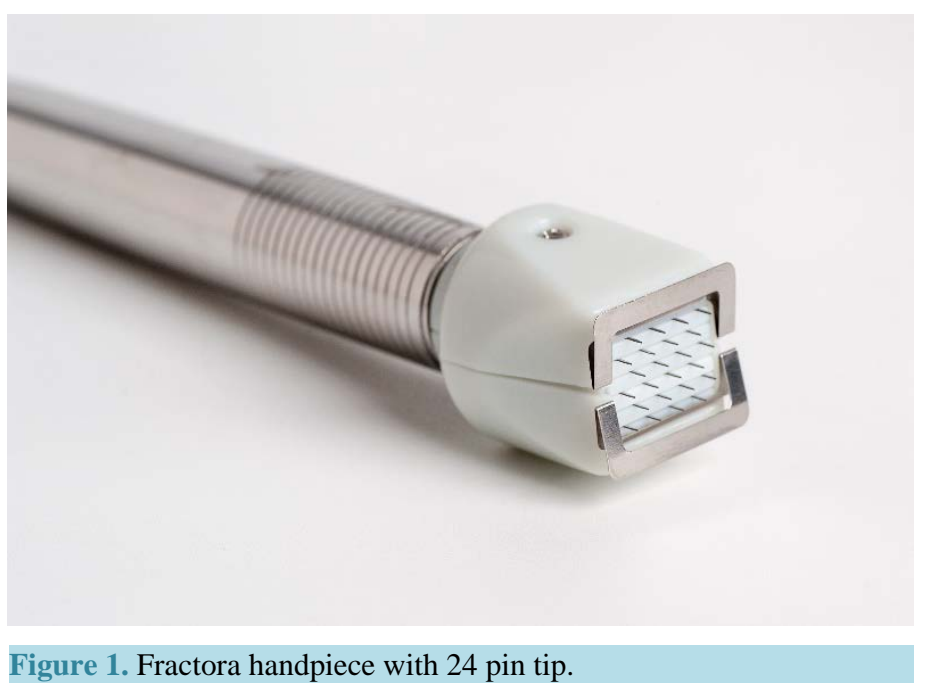

Outcome measurements: before and after pictures were qualitatively evaluated and compared for scar improvement and acne improvement. Representative skin biopsies from two patients were taken before and after treatment and examined with standard hematoxylin and eosin (H\&E) stain as well as Verhoeff's Van Gieson (VVG) and Shikata stains for elastic fibers.

\section{Results}

We identified eight patients who completed four treatments (Table 1), six females and two males with a median age of 20.5 years (range 17 - 41). All patients demonstrated significant improvement of active acne lesions and acne scarring (Figure 2).

Histological sections of skin biopsies taken before treatment demonstrate long dermal scars $1.5 \mathrm{~mm}$ deep (Figure 3(a)) that are characterized by thick disorganized collagen fibers (Figure 3(b)) and lack of adnexal structures, such as hair follicles and sebaceous glands (Figure 3(a)), as well as lack of elastic fibers (Figure $3(\mathrm{c}))$.

Treatment outcome after four Fractora treatments is expressed in the reduction of scar depth to about half (Figure 4(a)) and appearance of new collagen fibers that pushed the scar upwards (Figure 4(b)). Repopulation of the scar area with adnexal structures that were devoid of the original scar tissue was also observed (Figure 4(a)). In addition, elastic fibers that were devoid of the original scar tissue repopulated in the treated area (Figure 4(c)).

\section{Discussion}

We report our experience with a fractional radiofrequency (FRF) device for the treatment of active acne lesions and acne scarring. Treatment with FRF led to significant improvement in the depth of the scars as demonstrated by significant improvement in clinical scoring and confirmed by pathological assay. Skin biopsies after FRF treatment demonstrated that scar thickness diminished in depth from 1.5 to $0.8 \mathrm{~mm}$, having regressed higher into the superficial dermis, pushed up by the new collagen fibers. In addition, there was repopulation of adnexal structures and new elastic fibers in the superficial dermis previously occupied by scar tissue (Figure 3, Figure 4). FRF delivered electrical energy to create zones of thermal damage to the dermis in the area treated while sparing adjacent areas which serve as a reservoir for wound healing [6]. The coated pin tips used in this study feature additional epidermal protection in darker skin patients. Combination of these properties reduce the damage to the skin and result in the desired outcome with minimal downtime. In addition, the needling effect of the pins may provide additional benefit for acne scar treatment [7].

RF is hypothesized to improve AV by sebum-suppression and its effect on AV has been demonstrated elsewhere [8]. In our cohort, significant resolution of AV lesions was observed. These results suggest that FRF may be used as a modality that allows for simultaneous treatment of both active acne lesions and previous scarring.

While the treatment itself involved some discomfort, via the pins inserted through the epidermis, the overall 


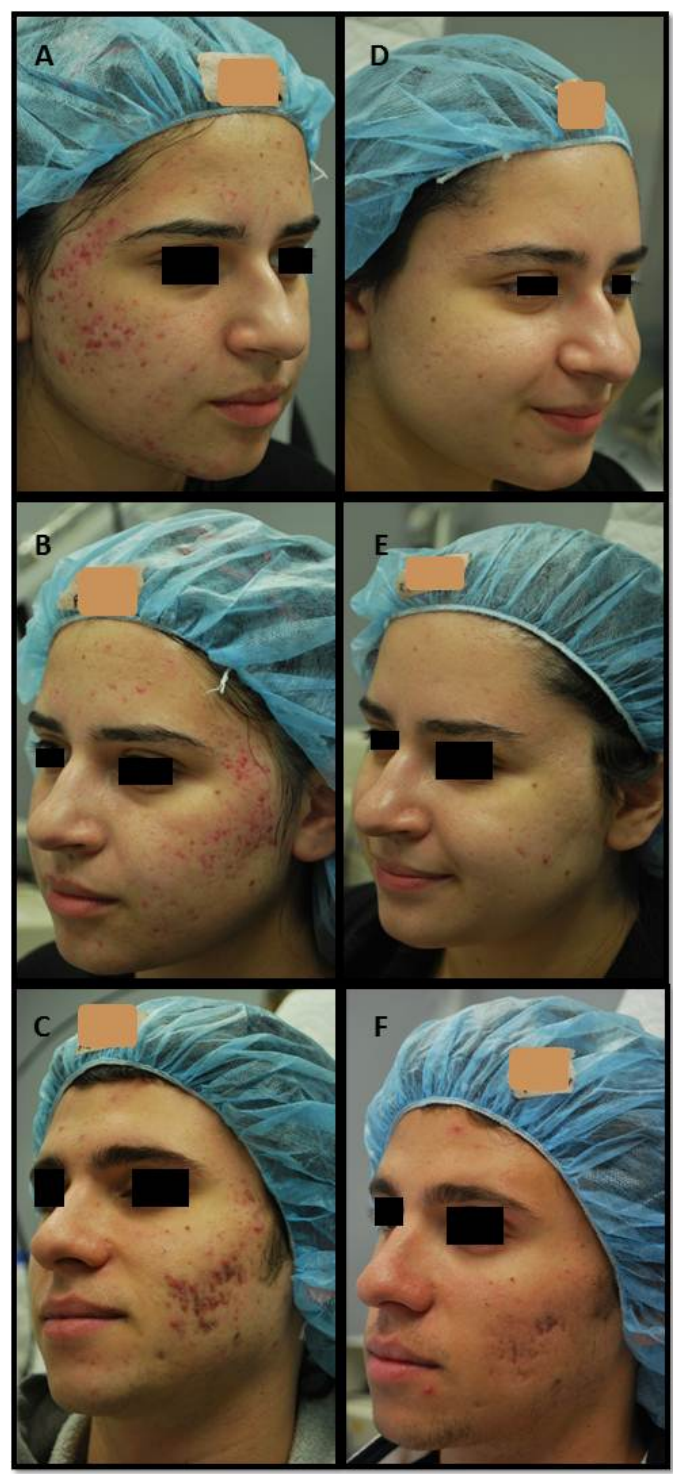

Figure 2. Active acne and acne scars before (A, B, C) and after (D, E, F) four Fractora treatments.

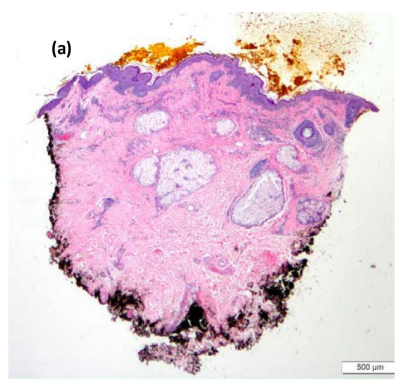

Acne scar tissue is $1.5 \mathrm{~mm}$ deep and devoid of hair $f$
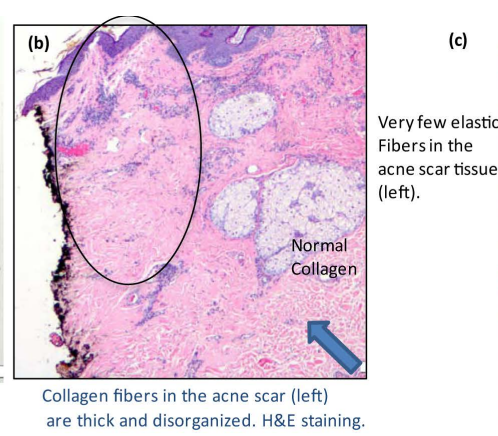

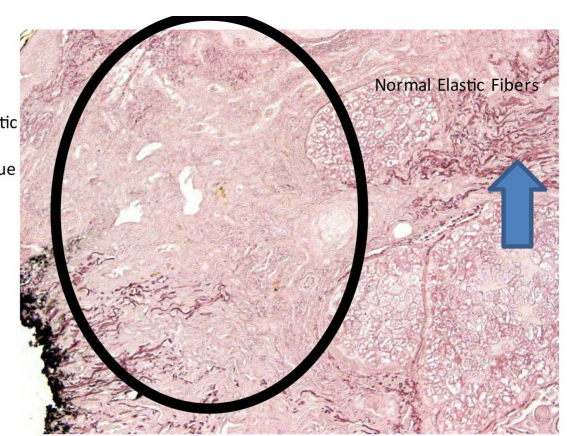

Figure 3. Histological sections of skin biopsies from a representative patient before Fractora treatments. (a) Demonstrates acne scar tissue $1.5 \mathrm{~mm}$ deep in the dermis, devoid of hair follicles and sebaceous glands. H\&E staining. (b) Demonstrates thick and disorganized collagen fibers in the acne scar tissue (circle), as opposed to normal collagen pointed by arrow. H\&E staining. (c) Demonstrates acne scar tissue mostly devoid of elastic fibers (circle), as opposed to normal elastic fibers pointed by arrow. VVG and Shikata staining. 


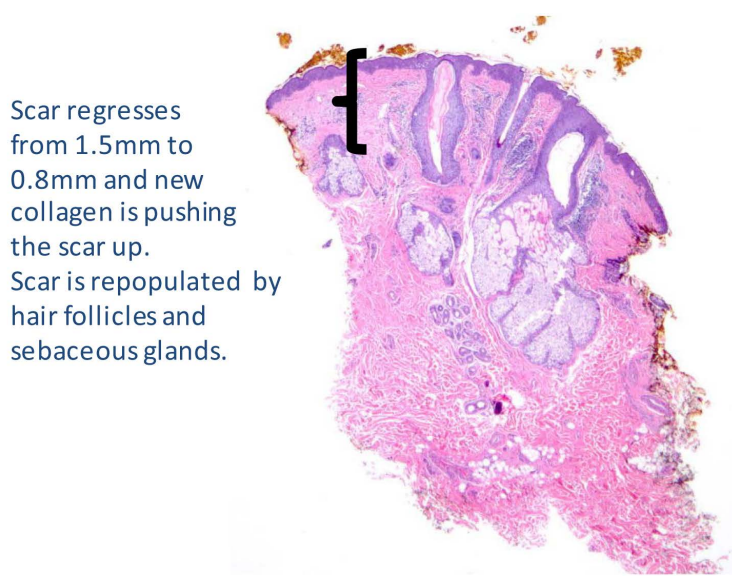

(a)

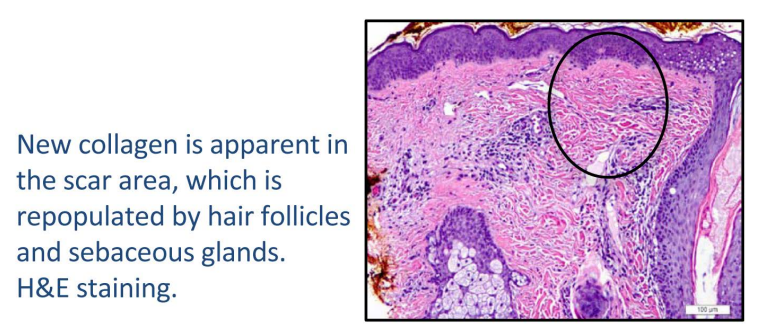

(b)

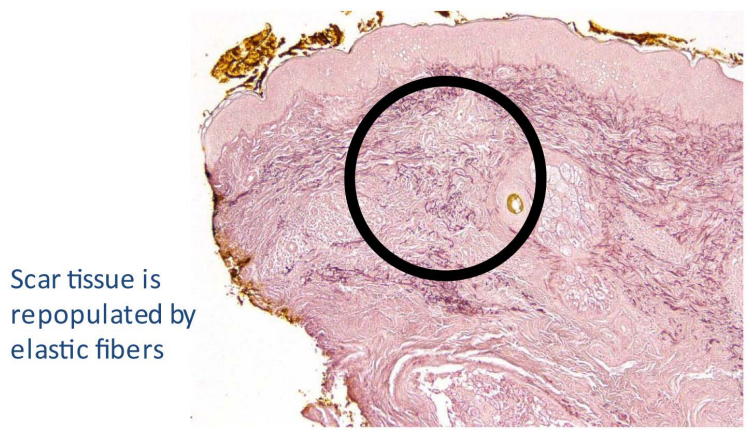

(c)

Figure 4. Histological sections of skin biopsies from a representative patient after four Fractora treatments. (a) Demonstrates reduction of scar depth to 0.8 $\mathrm{mm}$ and repopulation of adnexal structures in the scar tissue. H\&E staining. (b) Demonstrates new collagen fibers that are pushing the scar upwards (circle) H\&E staining. (c) Demonstrates repopulation of the papillary dermal scar area with elastic fibers (circle). VVG and Shikata staining.

patient experience was positive without any significant adverse events reported. Some patients noted red needle marks and/or sunburn like erythema for up to 48 hours.

For the purpose of this study we arbitrarily decided on a four treatment regimen and while some patients achieved satisfactory response in four treatments, we have excluded patients with satisfactory response with fewer treatments. Other patients elected to add more treatments and we therefore conclude the total number of treatments required can be variable and depends on the goals of treatment and the patient's individual response rate.

FRF treatment has significant advantages as it is minimally invasive, has very low downtime (most patients required 3 - 4 days of recovery) and safe. We did not encounter any cases of pigment alteration and in fact can now report anecdotal use of coated tips in treatment of Fitzpatrick skin type 6 patients without adverse events (data not shown). This may be of significant value as adult acne and atrophic scarring may be more prevalent in 
darker skin types [9]. In addition, we have anecdotally noted some skin tightening in our patients which is a positive unintended outcome that is well received among our older patients.

Of note, our study has a few limitations since it is a single practitioner experience and it is a retrospective case series. The treatment involved significant discomfort, but the company has redesigned the pulse configuration to reduce the pain considerably. The treatment is not covered by most insurance providers.

\section{Conclusion}

In conclusion, FRF emerges as a safe and effective treatment modality for AV and acne related scars. Further randomized controlled studies are required to fully evaluate the magnitude of this positive effect and more basic science studies are needed in order to better characterize its mechanism of action on acne lesions.

\section{References}

[1] Rivera, A.E. (2008) Acne Scarring: A Review and Current Treatment Modalities. Journal of the American Academy of Dermatology, 59, 659-676. http://dx.doi.org/10.1016/j.jaad.2008.05.029

[2] Thomas, D.R. (2004) Psychosocial Effects of Acne. Journal of Cutaneous Medicine and Surgery, 8, 3-5. http://dx.doi.org/10.1007/s10227-004-0752-x

[3] Del Rosso, J.Q. and Kircik, L.H. (2013) The Sequence of Inflammation, Relevant Biomarkers, and the Pathogenesis of Acne Vulgaris: What Does Recent Research Show and What Does It Mean to the Clinician? Journal of Drugs in Dermatology: JDD, 12, s109-s115.

[4] Fabbrocini, G., Annunziata, M.C., D’Arco, V., et al. (2010) Acne Scars: Pathogenesis, Classification and Treatment. Dermatology Research and Practice, 2010, Article ID: 893080. http://dx.doi.org/10.1155/2010/893080

[5] Goodman, G.J. (2011) Treatment of Acne Scarring. International Journal of Dermatology, 50, 1179-1194. http://dx.doi.org/10.1111/j.1365-4632.2011.05029.x

[6] Lolis, M.S. and Goldberg, D.J. (2012) Radiofrequency in Cosmetic Dermatology: A Review. Dermatologic Surgery, 38, 1765-1776. http://dx.doi.org/10.1111/j.1524-4725.2012.02547.x

[7] Alam, M., Han, S., Pongprutthipan, M., et al. (2014) Efficacy of a Needling Device for the Treatment of Acne Scars: A Randomized Clinical Trial. JAMA Dermatology, 150, 844-849. http://dx.doi.org/10.1001/jamadermatol.2013.8687

[8] Ruiz-Esparza, J. and Gomez, J.B. (2003) Nonablative Radiofrequency for Active Acne Vulgaris: The Use of Deep Dermal Heat in the Treatment of Moderate to Severe Active Acne Vulgaris (Thermotherapy): A Report of 22 Patients. Dermatologic Surgery, 29, 333-339; discussion 9.

[9] Perkins, A.C., Cheng, C.E., Hillebrand, G.G., Miyamoto, K. and Kimball, A.B. (2011) Comparison of the Epidemiology of Acne Vulgaris among Caucasian, Asian, Continental Indian and African American Women. Journal of the European Academy of Dermatology and Venereology: JEADV, 25, 1054-1060. http://dx.doi.org/10.1111/j.1468-3083.2010.03919.x

[10] Fitzpatrick, T.B. (1988) The Validity and Practicality of Sun-Reactive Skin Types I through VI. Archives of Dermatology, 124, 869-871. http://dx.doi.org/10.1001/archderm.1988.01670060015008 